\title{
Transfer of clinically relevant gene expression signatures in breast cancer: from Affymetrix microarray to Illumina RNA-Sequencing technology
}

\author{
Debora Fumagalli ${ }^{1 \dagger}$, Alexis Blanchet-Cohen ${ }^{2 \dagger}$, David Brown ${ }^{1 \dagger}$, Christine Desmedt $^{1}$, David Gacquer ${ }^{3}$, \\ Stefan Michiels ${ }^{4,5}$, Françoise Rothé ${ }^{1}$, Samira Majjaj ${ }^{1}$, Roberto Salgado ${ }^{6}$, Denis Larsimont ${ }^{7}$, Michail Ignatiadis ${ }^{1}$, \\ Marion Maetens ${ }^{1}$, Martine Piccart ${ }^{6}$, Vincent Detours ${ }^{3}$, Christos Sotiriou ${ }^{1 *+}$ and Benjamin Haibe-Kains $8^{8, *^{*}}$
}

\begin{abstract}
Background: Microarrays have revolutionized breast cancer $(\mathrm{BC})$ research by enabling studies of gene expression on a transcriptome-wide scale. Recently, RNA-Sequencing (RNA-Seq) has emerged as an alternative for precise readouts of the transcriptome. To date, no study has compared the ability of the two technologies to quantify clinically relevant individual genes and microarray-derived gene expression signatures (GES) in a set of BC samples encompassing the known molecular BC's subtypes. To accomplish this, the RNA from 57 BCs representing the four main molecular subtypes (triple negative, HER2 positive, luminal A, luminal B), was profiled with Affymetrix HG-U133 Plus 2.0 chips and sequenced using the Illumina HiSeq 2000 platform. The correlations of three clinically relevant BC genes, six molecular subtype classifiers, and a selection of 21 GES were evaluated.

Results: 16,097 genes common to the two platforms were retained for downstream analysis. Gene-wise comparison of microarray and RNA-Seq data revealed that $52 \%$ had a Spearman's correlation coefficient greater than 0.7 with highly correlated genes displaying significantly higher expression levels. We found excellent correlation between microarray and RNA-Seq for the estrogen receptor (ER; $\left.r_{s}=0.973 ; 95 \% \mathrm{Cl}: 0.971-0.975\right)$, progesterone receptor ( $\mathrm{PgR} ; r_{\mathrm{s}}=0.95$; 0.947-0.954), and human epidermal growth factor receptor 2 (HER2; $r_{s}=0.918 ; 0.912-0.923$ ), while a few discordances between ER and PgR quantified by immunohistochemistry and RNA-Seq/microarray were observed. All the subtype classifiers evaluated agreed well (Cohen's kappa coefficients $>0.8$ ) and all the proliferation-based GES showed excellent Spearman correlations between microarray and RNA-Seq (all $r_{s}>0.965$ ). Immune-, stroma- and pathway-based GES showed a lower correlation relative to prognostic signatures (all $r_{s}>0.6$ ).
\end{abstract}

Conclusions: To our knowledge, this is the first study to report a systematic comparison of RNA-Seq to microarray for the evaluation of single genes and GES clinically relevant to BC. According to our results, the vast majority of single gene biomarkers and well-established GES can be reliably evaluated using the RNA-Seq technology.

Keywords: Breast cancer, Gene expression signatures, Affymetrix, Microarray, Illumina, RNA-Seq, Immunohistochemistry, Estrogen receptor, Progesterone receptor, HER2 receptor

\footnotetext{
* Correspondence: christos.sotiriou@bordet.be; bhaibeka@uhnresearch.ca

${ }^{\dagger}$ Equal contributors

'Breast Cancer Translational Research Laboratory (BCTL), Institut Jules Bordet,

Brussels, Belgium

${ }^{8}$ Princess Margaret Cancer Centre, University Health Network, Toronto, ON,

Canada

Full list of author information is available at the end of the article
}

\section{() Biomed Central}

(c) 2014 Fumagalli et al.; licensee BioMed Central Ltd. This is an Open Access article distributed under the terms of the Creative Commons Attribution License (http://creativecommons.org/licenses/by/4.0), which permits unrestricted use, distribution, and reproduction in any medium, provided the original work is properly credited. The Creative Commons Public Domain Dedication waiver (http://creativecommons.org/publicdomain/zero/1.0/) applies to the data made available in this article, unless otherwise stated. 


\section{Background}

For more than a decade, microarrays have represented the most comprehensive approach to measuring gene expression levels [1]. Their ability to simultaneously assess thousands of transcripts, coupled with relatively low experimentation costs and the broad availability of analytical tools, have facilitated their wide use and led to fundamental advances in several research fields. In breast cancer, implementing gene expression microarrays has broadened our knowledge about the biology of the disease, which has, for many years, relied on immunohistochemistry (IHC) and clinical-pathologic parameters only. Several studies have shown that breast cancers can be classified into at least four "intrinsic" subtypes (basal-like, HER2 enriched, luminal A, and luminal B) which can only be partially recapitulated by IHC definitions of the three fundamental breast cancer biomarkers: estrogen receptor (ER), progesterone receptor (PgR), and human epidermal growth factor receptor 2 (HER2) [2-5].

In current clinical practice, subtypes are defined mostly by using an IHC surrogate [6]. Multiple expression-based classifiers have been developed, including two versions of the Subtype Classification Model (SCM) (SCMOD1 [7] and SCMOD2 [8]), and the simple three-gene model (SCMGENE [9]) developed by Sotiriou and co-workers, as well as three variants of the Single Sample Predictor (SSP) (SSP2003 [10], SSP2006 [11] and PAM50 [12]) developed by Perou and co-workers, the latter having been recently translated into a clinical assay (Prosigna" [13]). The computational implementation and comparison of these classifiers has been documented in [9]. Microarrays have also been used to derive a series of gene expression signatures aimed at characterizing the biology of the disease and at helping clinicians predict relapse and response to treatment more accurately than tools using traditional clinico-pathological parameters [7,12,14-27]. Some of these signatures have been endorsed by international breast cancer guidelines, and they are being increasingly implemented in standard practice [6].

In parallel, RNA sequencing (RNA-Seq) is emerging as the technology of reference for thorough characterization of the human transcriptome and as a superior alternative to microarrays to define gene expression levels [28-31]. RNA-Seq is overcoming some of the drawbacks of microarrays [28-32]. For instance, the dependence of microarrays on hybridization of transcripts to pre-determined probes restricts analysis to genes for which genomic sequence information is available first-hand and to sequences that are distant enough so that probes do not cross-hybridize. Moreover, high levels of background noise arising from non-specific hybridization and probe saturation affect the quantification of transcripts expressed at low and high levels, respectively, limiting the dynamic range of the technology. Although the RNA-Seq technology efficiently address these issues, the current lack of standards for analyzing these new data, coupled with the relatively high cost of the RNA-Seq experiments, could deter investigators from implementing the technology in their activity.

Several studies have been carried out to compare the performance of RNA-Seq and microarrays, including exon arrays, in defining levels of gene expression [33-42]. The vast majority of them have focused on establishing the reliability of RNA-Seq in differential gene expression (DGE) analyses between two or more samples and/or conditions of interest. Despite the fact that they have generally reported good correlation between the two technologies, most of these studies have relied on relatively few samples or exclusively on non-human samples. Moreover, they have never attempted to assess the performance of RNA-Seq in defining clinically relevant biomarkers developed using microarrays. Given the promise of microarray-based gene signatures [43] and the significant advantages of the new RNA-Seq technology in providing more accurate and reliable gene expression measurements, there is a dire need to investigate the transition of breast cancer gene signatures from microarray to RNA-Seq.

The main aim of the current study is to compare the agreement between two of the most widely used microarray and RNA-Seq platforms, Affymetrix and Illumina HiSeq respectively, in estimating (1) the expression of single genes which are clinically relevant to breast cancer and (2) breast cancer subtype classifiers and gene expression signatures that have been developed over the years with microarrays. The comparison uses a dataset obtained from well characterized breast cancer patients representing the four main breast cancer subtypes: triple negative, HER2 positive, luminal A, and luminal B.

\section{Methods}

\section{Sample selection and characterization}

Fresh-frozen tumor material was obtained from 57 breast cancer patients who were treated at Institut Jules Bordet (Brussels, Belgium) between 2007 and 2011 and whose samples were stored at the institute's biorepository. The samples represented the four main known breast cancer subtypes (by IHC) and their tumor cell content evaluated on a hematoxylin-eosin (H\&E) slide by a board-certified breast cancer pathologist was greater than or equal to $30 \%$. Of the 57 patients, 17 had triple negative breast cancer (TN: ER, PgR, and HER2 negative), 14 HER2 positive (any ER and PgR, HER2 positive), 16 luminal A (ER, HER2 negative, histological grade 1), and 10 luminal B (ER, HER2 negative, histological grade 3). The use of the tumor material is consistent with the informed consent signed by the patients and was granted approval by Institut Jules Bordet's ethics committee (approval number: 
CE1967), and is in accordance with the applicable laws and regulations in Belgium.

\section{RNA extraction}

RNA was extracted using TRIzol ${ }^{\circ}$ (Life Technologies, Carlsbad, California) following the manufacturer's instructions. Concentration was measured using the NanoDrop 1000 (Thermo Scientific, Waltham, Massachusetts), and integrity (RIN: RNA Integrity Number) was assessed using an Agilent 2100 Bioanalyzer (Agilent Technologies, Santa Clara, California). All the samples yielded enough material for downstream experiments, and had a RIN equal to or greater than 6.5. RNA obtained from the same extraction procedure was profiled on microarrays and sequenced on the Illumina HiSeq 2000.

\section{Microarray experiments}

100 ng of total RNA was profiled at the Institut Jules Bordet using the Affymetrix ${ }^{\circ}$ HG-U133 Plus 2.0 Arrays (Affymetrix, Santa Clara, California), following the manufacturer's instructions. Briefly, the RNA was first reversetranscribed into double-stranded cDNA. This cDNA was transcribed in vitro. After purification of the aRNA, $12.5 \mu \mathrm{g}$ were fragmented and labeled prior to hybridization to the chips. Quality control (QC) for each chip was performed following the recommendations posted on [44]. Following $\mathrm{QC}$, the probe level intensities were background adjusted and quantile normalized using the Frozen Robust Multiarray Analysis (fRMA) method [45] as implemented in the R/Bioconductor package fmra [46]. Probeset level annotations were obtained from $\mathrm{R} /$ Bioconductor package jetset [47] and complemented with BioMart [48]; when multiple probesets mapped to the same Entrez Gene ID, the probeset with the highest jetset score was selected. The raw Affymetrix CEL files are available from the NCBI's Gene Expression Omnibus under accession number GSE43358.

The data can be accessed through this link: http:// www.ncbi.nlm.nih.gov/geo/query/acc.cgi? token=trmzbecoaqyugtc\&acc $=$ GSE43358.

\section{RNA-Seq sample preparation and sequencing}

Transcriptome sequencing was performed at DNAVision (Gosselies, Belgium). Transcriptome libraries were constructed using the Illumina ${ }^{-}$TruSeq $^{\text {ma }}$ RNA Sample Preparation Kit for paired-end reads sequencing on the HiSeq 2000 (Illumina, San Diego, California) following the manufacturer's instructions. Briefly, starting from $1 \mu \mathrm{g}$ of total RNA, the poly-A containing mRNA molecules were purified using poly- $\mathrm{T}$ oligo-attached magnetic beads. Following purification, the mRNA was fragmented into small pieces using divalent cations under elevated temperature. The cleaved RNA fragments were copied into first strand cDNA using reverse transcriptase and random primers. This was followed by second strand cDNA synthesis using DNA Polymerase I and RNase $\mathrm{H}$ and purification using the AMPure XP beads (Agencourt BioSciences Corporation, Beverly, Massachusetts). The cDNA fragments were end repaired with the addition of a single ' $A$ ' base and the ligation of adapters. The products were purified using the AMPure XP beads and enriched with PCR (15 cycles) to create the final cDNA library followed by purification using the AMPure XP beads. Library quality control and quantification were performed using the Agilent Bioanalyser 2100 and qRTPCR. The libraries were then pooled (4 libraries/pool). Clusters were generated in a cBot Cluster Generation System using the Paired-End Cluster Generation Kit v2HS and sequenced on the Illumina ${ }^{\circ}$ HiSeq 2000 platform with a $2 \times 50$ base-pairs paired-end mode. Base calls were made using the Illumina CASAVA 1.5 pipeline. Sequence data has been deposited at the European Genome-phenome Archive (EGA), which is hosted by the EBI and the CRG [49], under study's accession number EGAS00001000495 and dataset's accession number EGAD00001000626.

\section{Assessment of RNA-Seq data quality}

The following statistics were computed to verify the quality of the RNA-Seq data (Additional file 1: Table S1). The total number of paired reads, the average Phred quality scores, and the average GC content were calculated on the quality trimmed FASTQ files with FastQC [50]. The percentage of "proper pairs", defined as mapped paired reads with an insert size ranging from 60 to $160 \mathrm{bp}$, was calculated with BamTools [51]. The percentage of aligned duplicate read pairs was calculated with Picard tools MarkDuplicates [52].

\section{RNA-Seq analysis}

After trimming the poor quality bases, the reads were mapped to the human reference genome hg19 with TopHat2 (version 2.0.0) [53], and gene expression was quantified with Cufflinks (version 2.0.0) [53]. The annotation file (GTF file) used for both alignment and gene quantification was downloaded from Ensembl (on 26 July, 2012). To match the log scale of gene expression measurements from the microarray data, the FPKM (Fragments Per Kilobase of transcript per Million mapped reads) values computed by Cufflinks were logtransformed using the following formula:

$$
X^{\prime}=\log 2(X+1)
$$

where $\mathrm{X}$ represents the FPKM value computed by Cufflinks, and $\mathrm{X}^{\prime}$ is the log-transformed expression value. 


\section{Gene expression signatures}

Our study focused on the following gene expression signatures: (1) six prognostic signatures (GENE70 [14], GENE21 [15], Genomic Grade Index (GGI [16]), Risk of Relapse-Score (ROR-S [12]), ENDOPREDICT [17], and CIN70 [18]); (2) two immune signatures (STAT1MODULE [7] and IRMODULE [19]); (3) three stromarelated signatures (PLAUMODULE [7], DCN [20], and STROMACD10 [21]); and (4) ten pathway related signatures (PIK3CA-GS [22], PTEN loss [23], IGF1 [24], AKT/mTOR [25], MAPK [26], SRC, RAS, MYC, E2F3, and beta-catenin [27]). We also evaluated six subtype classifiers: SCMOD1 [7], SCMOD2 [8], SCMGENE [9], SSP2003 [10], SSP2006 [11], and PAM50 [12]. In addition to these multivariate subtyping models, we evaluated the three individual breast cancer clinically relevant genes: ER, PgR, and HER2.

We used the original signature algorithms for GENE70, GENE21, GGI, ROR-S, ENDOPREDICT, CIN70, STAT1MODULE, PLAUMODULE, DCN and STROMACD10 as implemented in the Bioconductor package genefu [54]. For the remaining gene expression signatures, we computed the signature scores following the approach used in Ignatiadis et al. [55]. The scores were computed from the list of genes in their respective signatures (as listed in GeneSigDB [56]) as the weighted average using the following formula:

$$
s=\frac{\sum_{i \subset n} w_{i} x_{i}}{\sum_{i \subset n}\left|w_{i}\right|}
$$

where $s$ is the signature score, $n$ is the number of genes in the signature of interest, $x_{i}$ is the expression of the gene, and the gene-specific weight $w_{i} \in\{-1,1\}$ is the sign of the coefficient defined in the original publication. Only genes that could be mapped to EntrezGene IDs were used. Finally, each signature score was rescaled so that the $2.5 \%$ and $97.5 \%$ quantiles were equal to +1 and -1 respectively.

\section{Data analysis}

The pair-wise correlation between Affymetrix microarrays and Illumina RNA-Seq gene expression data and gene expression signatures scores was assessed using Spearman's rank-based correlation. For the three single gene biomarkers (ER, PgR and HER2), the correlation between microarray or RNA-Seq with IHC was estimated to identify which technology provided better concordance with IHC. Cohen's kappa coefficient was used to compare the subtype classifications from microarray or RNA-Seq data. To statistically compare the Spearman correlation and Cohen's kappa coefficients of different gene signatures, we used a two-sided Wilcoxon rank sum test with 100 bootstrap replicates of the 57 patients to determine the p-value. The resulting p-values, reporting the significance of the correlation difference between each pair of gene expression signatures, were corrected for multiple testing using Bonferroni's method.

To compare the correlation of gene expression over the whole transcriptome between each pair of data type from a given sample, we used Spearman's rank-based correlation, the null distribution of which was established as the range of coefficients observed from all possible combinations of the 57 pairs excluding self-self pairs. This is efficiently computed from the cross correlation matrix minus the diagonal elements. The analyses performed in this study are fully reproducible and comply with proposed guidelines in terms of availability of the code and data [57]. The R scripts developed for the analysis are available upon request.

\section{Data availability}

The raw Affymetrix CEL files are available from the NCBI's Gene Expression Omnibus under accession number GSE43358. The data can be accessed through this link: http://www.ncbi.nlm.nih.gov/geo/query/acc.cgi? token $=$ trmzbecoaqyugtc\&acc $=$ GSE43358.

Sequence data has been deposited at the European Genome-phenome Archive (EGA), which is hosted by the EBI and the CRG [49], under study's accession number EGAS00001000495 and dataset's accession number EGAD00001000626.

\section{Results}

Gene-wise comparison of expression levels using Affymetrix microarray and Illumina RNA-Seq platforms

A subset of 16,097 genes were defined as common to the two platforms and retained for downstream analysis. Gene identifiers did not perfectly overlap due to differences in the annotation systems: jetset matched the Affymetrix probesets to the NCBI RefSeq human cDNA database, while the RNA-Seq analysis pipeline used Ensembl gene annotations (see Methods for more detail). When comparing the expression levels of the genes retained after selection of the best Affymetrix probeset, we found that although the scale of expression values differs due to different technology and normalization procedures, their rank is well conserved with $52 \%, 34 \%$, and $11 \%$ of these genes having Spearman's rank-based correlation greater than $0.7,0.8$, and 0.9 , respectively (Figure 1A). The Spearman correlation coefficient for each evaluated gene is reported in Additional file 1 : Table S2.

We observed that genes with the highest correlation coefficients for the comparison of microarray and RNASeq were significantly more expressed. Similarly, the 

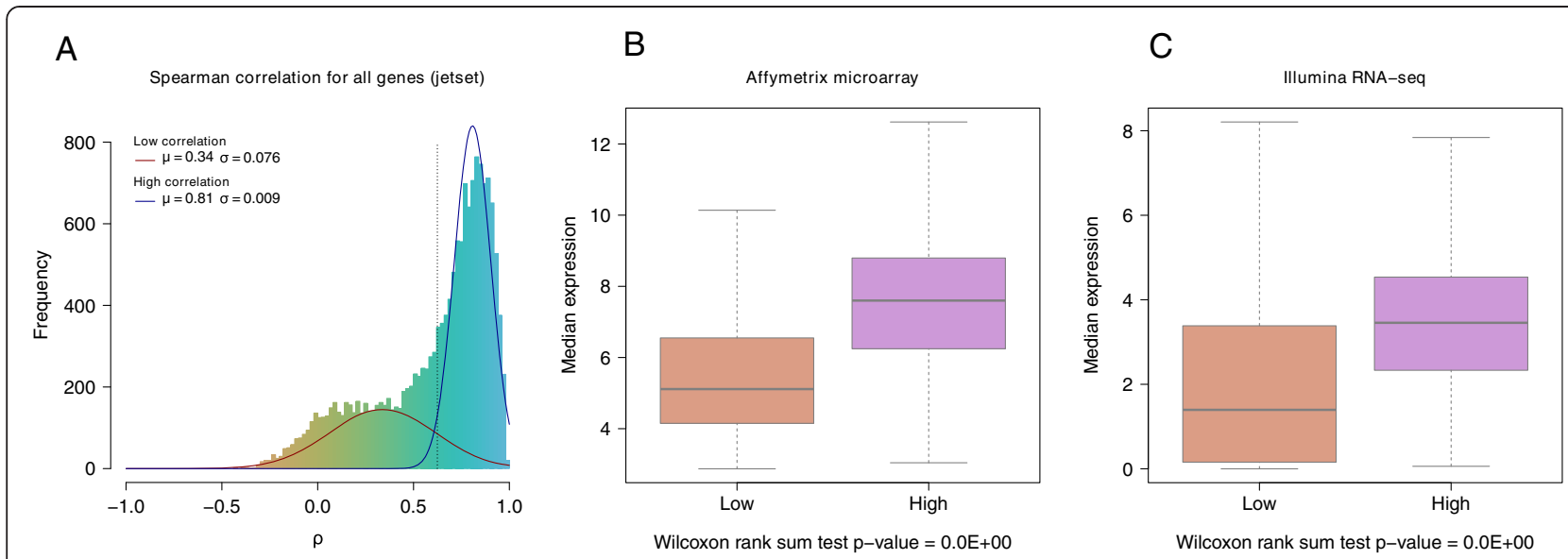

Figure 1 Gene expression correlation between Affymetrix microarray and Illumina RNA-Seq platforms. A: Expression correlation of the 16,097 genes measured both on Affymetrix microarray and Illumina RNA-Seq platforms after selecting the best Affymetrix probeset using jetset. B: and C: Box plots showing median level of gene expression for both Affymetrix and RNA-Seq for the genes with low $(<0.7)$ and high $(\geq 0.7)$ correlation. Genes highly correlated between the two platforms showed higher levels of expression than those with low correlation.

genes that were positively correlated were significantly more expressed than genes that were negatively correlated (two-sided Wilcoxon rank sum test $\mathrm{p}$-value $<1 \times 10^{-16}$; Additional file 2: Figure S1). This result holds true when considering a high cutoff of correlation $r_{s} \geq 0.7$ (two-sided Wilcoxon rank sum test $\mathrm{p}$-value $<1 \times 10^{-16}$; Figure $1 \mathrm{~B}$ and $1 \mathrm{C}$ ). This phenomenon could be explained by the potentially higher variance of genes expressed at low levels [58] or by the fact that microarrays have a limited dynamic range compared to RNA-Seq [28-32].

In order to investigate this phenomenon more deeply, we computed the correlation for genes with cumulative increasing expression (Additional file 3: Figure S2). By starting with genes expressed at low levels and increasing gene expression levels by increments of $1 \%$, we observed a steep rise in the median correlation coefficient; in contrast, the magnitude of the decrease moving away from genes expressed at high levels was much lower. These results suggest that, despite the potential saturation of microarrays for highly expressed genes, the correlation between the two technologies remains high; however, we observed a high-level of inconsistency for genes expressed at low levels, either originating from microarray or RNAseq technology, or both.

We acknowledge from past experience with gene expression microarrays that, when comparing the whole transcriptome, two unrelated samples may have a correlation coefficient that is as high as two arrays performed on the same sample, raising questions about the significance of an asymptotic p-value in that particular setting. In our dataset, the correlation of gene expression profiles for the whole transcriptome measured by microarray and RNA-Seq was statistically significant for all except three patients (Additional file 4: Figure S3).

\section{Definition of ER, PgR, and HER2 status according to IHC, microarray, and RNA-Seq}

Among the genes retained for analysis, we focused our attention on three that are clinically relevant for breast cancer: ER, PgR, and HER2. Measuring them precisely is of utmost importance to clinical practice as these are presently the only validated breast cancer predictive biomarkers available, and they are routinely used to make decisions about patient treatment $[6,59]$.

When comparing the expression levels of these three genes as defined by microarray and RNA-Seq, we found excellent Spearman correlation coefficients: 0.973 for ER [95\% CI: 0.971-0.975]; 0.95 for PgR [95\% CI: 0.947-0.954]; and 0.918 for HER2 [95\% CI: 0.912-0.923] (Figure 2).

We then went a step further and compared the gene expression levels defined by either RNA-Seq or Affymetrix with IHC, which is currently considered to be the methodology of reference for the definition of these markers, together with FISH for HER2 [60,61]. Overall, a good correlation was found between the technologies $\left(r_{s}>0.69\right)$, and only a few discordances were observed (Additional file 5: Figure S4).

\section{Correlation between technologies for the definition of breast cancer subtype classifiers}

Two different gene expression approaches have been developed to prospectively classify breast cancers into molecular subtypes: Subtype Classification Models (SCMs) [7-9] and Single Sample Predictors (SSPs) [10-12], which include PAM50. In the current dataset, 

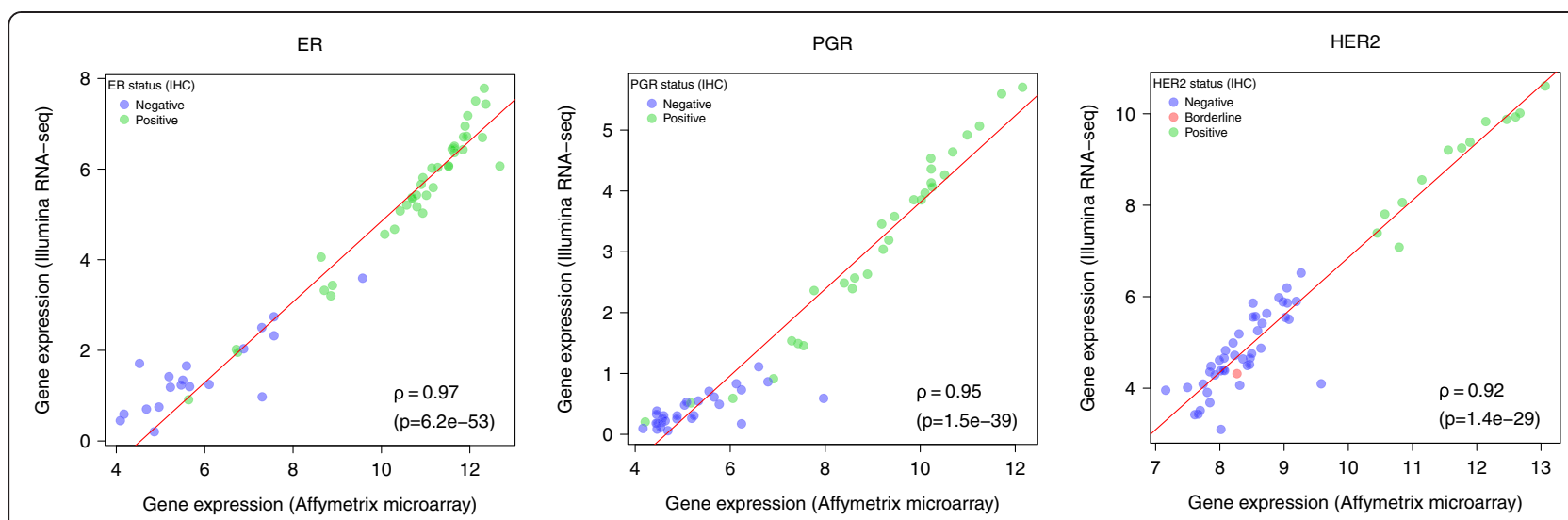

Figure 2 Expression correlation for ER, PgR, and HER2 genes. Scatterplots reporting the expression correlation of ER, PgR, and HER2 defined by Affymetrix microarray or Illumina RNA-Seq. Each dot is colored according to the corresponding status determined by IHC: green for positive, blue for negative, red for borderline. Spearman correlation coefficient and p-value are provided below the plots.

our subtype classifier SCMOD2 [8] showed the highest correlation between microarray and RNA-Seq technologies $(\kappa=0.975$; Figure 3A, Additional file 1: Table S3), which was significantly higher than the other classifiers (100 bootstrap replicates, corrected p-value <0.001; Additional file 1: Table S4). Of note, although the kappa coefficients for SCMGENE [9] and PAM50 [12] were very similar ( $\kappa=0.903$ vs. 0.902 for SCMGENE and PAM50, respectively), SCMGENE was more concordant than PAM50 in our study (corrected p-value $=0.001$, Additional file 1: Table S4).

\section{Correlation between technologies: gene prognostic} signatures

Using microarray technology, several prognostic gene expression signatures have been developed in the

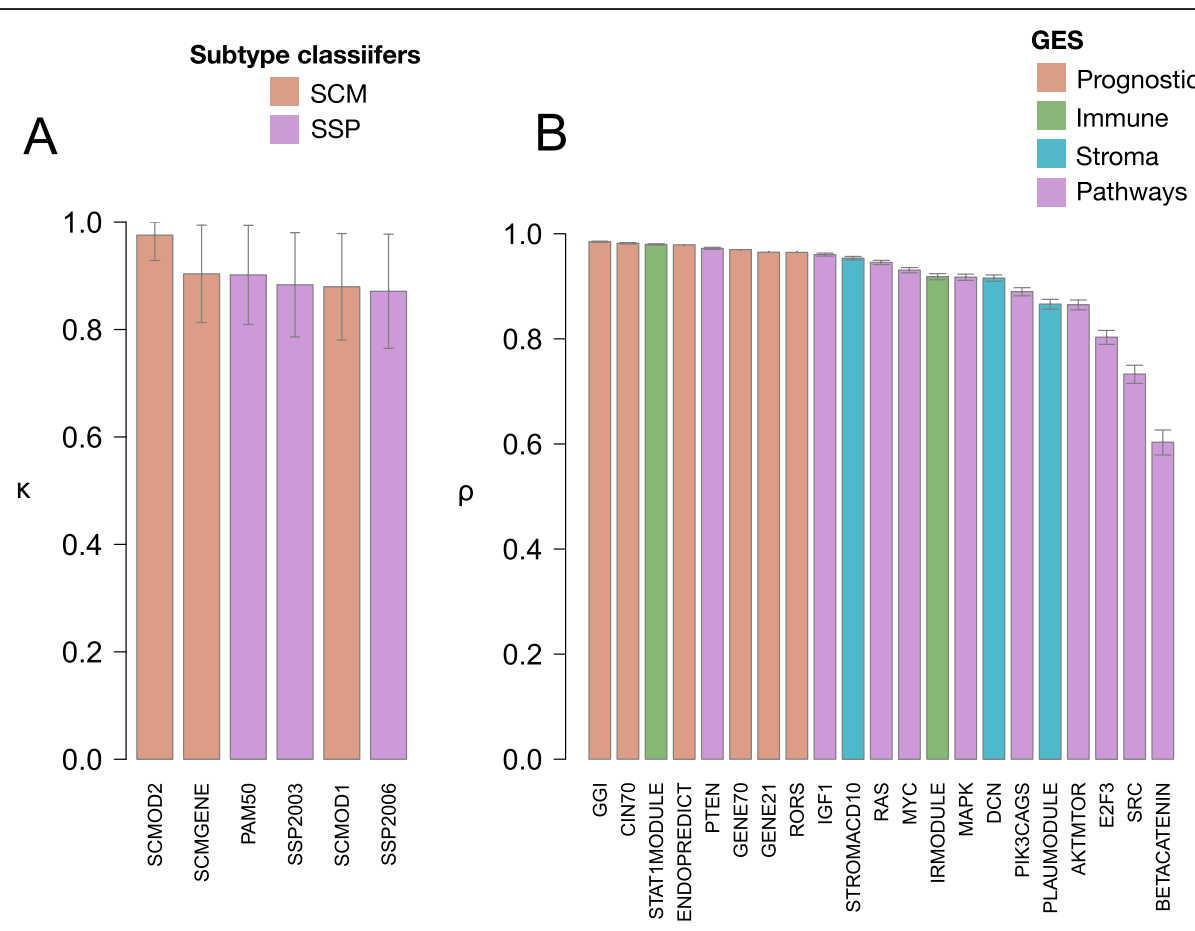

Figure 3 Correlation values for the evaluated subtype classifiers and gene expression signatures. A: Cohen's Kappa coefficients for subtype classifiers (orange: SCMs; purple: SSPS). B: Spearman correlation values for prognostic (orange), immune (green), stroma (blue) and pathway (purple) signature scores as computed using Affymetrix microarray and Illumina RNA-Seq platforms. 
attempt to help clinicians to identify which breast cancers are at high or low risk of recurrence [43]. Among these, MammaPrint ${ }^{\circ}$ (here referred to as GENE70) [14], OncotypeDx ${ }^{\circ}$ (here referred to as GENE21) [15], GGI [16], ENDOPREDICT [17] and ROR-S [12] have been widely investigated and applied in the clinical setting. When comparing the values of these signatures on a continuum as defined by either microarray or RNA-Seq, an excellent Spearman correlation was found: 0.97 [95\% CI 0.968-0.972] for GENE70; 0.965 [95\% CI 0.962-0.967] for GENE21; 0.985 [95\% CI 0.984-0.986] for GGI; 0.979 [95\% CI 0.977,0.981] for ENDOPREDICT; and 0.965 (95\% CI $0.962,0.967$ ) for ROR-S (Figure 4).

\section{Correlation between technologies: immune, stroma and pathway related gene expression signatures}

After a first wave of prognostic signatures, which essentially captured tumor proliferation signals, a new generation of "biological" signatures were developed that focused on determining the prognostic and/or predictive role of host-tumor immune interplay, tumor microenvironment and pathway activation signaling.
We evaluated the correlation between microarray and RNA-Seq platforms in measuring the following: 1) two immune signatures (STAT1MODULE [7], IRMODULE [19]); 2) three stroma signatures (PLAUMODULE [7], DCN [20], STROMACD10 [21]); and 3) ten pathway signatures (PIK3CA-GS [22], PTEN loss [23], IGF1 [24], AKT/mTOR [25], MAPK [26], SRC, RAS, MYC, E2F3, beta-catenin [27]).

As shown in Figure 3B, the Spearman correlation coefficients were better overall for the prognostic signatures than the biological ones (corrected p-value $<0.001$ ). This was particularly the case for the pathway signatures.

\section{Signature enrichment in highly expressed genes and correlation between technologies}

Since higher correlation coefficients were found for genes expressed at higher levels, we investigated whether enrichment in genes with higher expression in the above subtype classifiers and gene expression signatures could have affected their correlation when defined with the two technologies. We found that $81 \%(22 / 27)$ and $74 \%(20 / 27)$ of the signatures were significantly enriched with highly

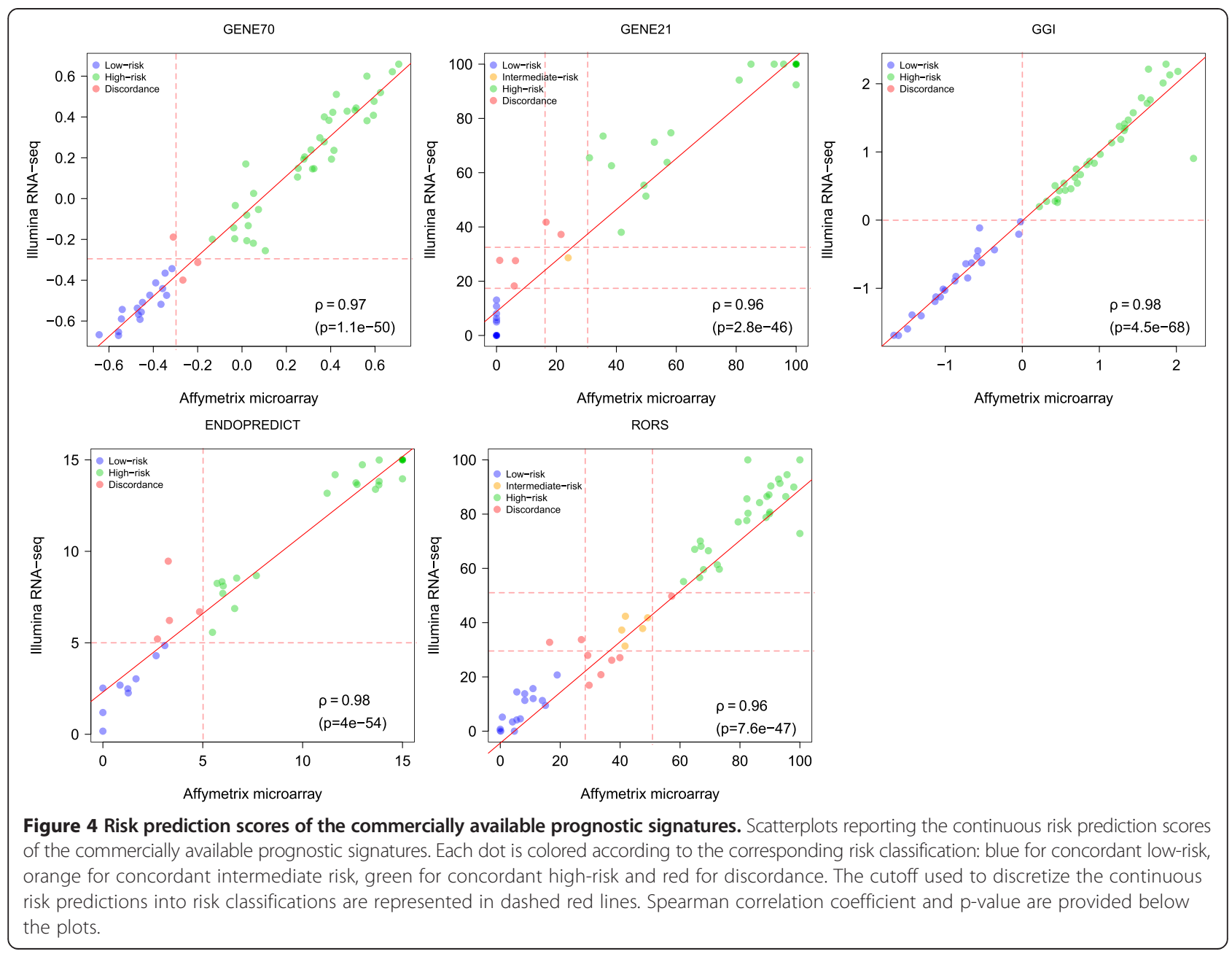


expressed genes for Affymetrix microarray and Illumina RNA-seq platforms, respectively (p-value $<0.05$; Additional file 6: Figure S5 and Additional file 7: Figure S6). However such enrichment did not imply significantly higher correlation between the two platforms, suggesting that the proportion of highly expressed genes is not solely responsible for the highest correlations observed in our study.

\section{Discussion}

The use of molecular tools in clinical practice is on the rise. In breast cancer, international guidelines endorse the implementation of microarray-derived gene signatures to support clinicians in the treatment decisionmaking process [6]. In addition, the upcoming results of two genome-forward clinical trials, namely the MINDACT and the TailorX trials $[62,63]$, involving thousands of patients, will provide the highest level of evidence to date about whether basing clinical decisions on microarrayderived prognostic gene signatures influences the outcome of breast cancer patients. The parallel rise of RNA-Seq as an accurate and reliable alternative to microarrays for transcriptome characterization and gene expression quantification is puzzling for investigators, who must decide which technology best fulfills their clinical needs. Hence, it is imperative to determine the reliability of transitioning from microarray to sequencing platforms in the clinical setting. To our knowledge, our is the first report investigating the correlation between the expression level of single clinically relevant genes and gene expression signatures obtained with the most commonly used microarray and RNA-Seq platforms, Affymetrix and Illumina respectively, in a selected dataset of breast cancer patients.

When focusing on the genes in common between the two platforms, our analysis showed that the expression of more than half of them had a Spearman correlation of 0.7 . This was the case simply by selecting the most specific Affymetrix probeset and correlating it to gene log2 transformed FPKM obtained at sequencing, without any further computation. In studies exploring the correlation of the two technologies in defining genes differentially expressed among samples and/or conditions, the correlation values of fold change ratios were reported to range from 0.55 to 0.85 [33-42]. Of note, these values are similar to or higher than the correlation observed across different microarray platforms [64]. In these same studies, RNA-Seq seemed to be more reliable than microarray in identifying differential gene expression: in several reports, a large proportion of genes identified as differentially expressed by RNA-Seq but not by microarray were in fact confirmed by qPCR $[33,34,65]$. RNA-Seq also seems more suitable than microarrays to quantify absolute gene expression levels when compared with mass spectrometry measurements [35].
As reported by other investigators $[28,35,65]$, we found that the correlation of genes was higher when their expression levels were higher. The reliance of microarrays on the presence of primers and probes limits their ability to measure extreme gene expressions; according to our data, the limitation seems to be more relevant in the low expression range. On the contrary, the nature of sequencing technologies allows the unbiased investigation of gene expression when enough depth is assured.

In our study, RNA-Seq showed high correlation with microarrays when measuring clinically relevant genes in breast cancer (i.e., ER, PgR, and HER2). Nevertheless, despite an overall good correlation, both technologies showed a few discordances when compared to IHC. Similar results have already been reported when comparing microarray with IHC $[66,67]$. These discordances might be partly attributed to the fact that IHC measures the expression of ER, PgR, and HER2 at the protein level, while both Affymetrix and RNA-Seq measure the expression levels of the corresponding mRNAs. Although mRNA and protein expression levels are not fully independent, one cannot exclude that post-transcriptional regulation might affect their correlation. Another factor that could account for these discordances is tumor heterogeneity. The expression levels of the three markers can in fact vary across different areas of the tumor. While the RNA profiled on microarray and sequenced on the Illumina platform was obtained from the same tumor area, the slides used for IHC staining were cut from a distinct area of the tumor lesion. The best way to capture the activity level of these receptors and their downstream pathways remains largely unclear. It is possible that the determination of ER, PgR, and HER2 status at the mRNA level could provide clinicians with a more reproducible, quantitative, and informative assessment of these markers $[68,69]$. For the time being, however, not enough evidence is available to recommend mRNA-based measurements for clinical practice.

Because of the clinical relevance of breast cancer subtypes, much attention has been paid to the performance of microarray-derived subtype classifiers. Some concerns have been raised about their reliability though. It has been shown that although every SSP can consistently identify molecular subtypes with different levels of survival, they do not reliably assign the same patients to the same molecular subtype [70]. Variation in gene expression data and classification algorithms could influence how samples are classified into each subtype. In our study, the best correlation between microarray and RNA-Seq was obtained for SCMOD2 [8], one of the subtype classifiers developed by our group. Similar correlation coefficients were obtained for PAM50 [12] and SCMGENE [9], with the latter showing slightly higher concordance. This result suggests the higher robustness of SCMGENE to small perturbations, which concurs 
with our recent robustness study [9]. However, it is unlikely that Prosigna", the recent clinical assay implementing PAM50 using the NanoString ${ }^{\bullet}$ platform, would suffer the same limitation since its analytical validity has been thoroughly assessed [13].

Given the increasing implementation of microarrayderived gene signatures in clinical practice, it is vital to determine if RNA-Seq could reliably be used to define them. When considering 21 of the most relevant signatures developed in recent years [7,12,14-27], we found that the correlation values for microarray and RNA-Seq for signature determination ranged from moderate to very strong. Correlation values were higher for prognostic signatures than biological signatures, independently from their enrichment in highly expressed genes. This suggests that proliferation, which drives prognostic signatures, is more robust and reproducible than signals coming from other biological processes and that complex signatures developed on microarrays might be less stable.

\section{Conclusions}

In our study, we have demonstrated that RNA-Seq can reliably be used to evaluate both the expression of clinically relevant single genes and well established gene expression signatures originally defined with microarray technology. Considering the advantages that RNA-Seq offers over microarray, such as its ability to explore larger sets of genes, it is envisaged that its application to wider datasets could even provide information relevant for de-novo classification of breast cancer or for the development of new prognostic and predictive signatures. With the cost of RNA-Seq experiments decreasing continuously and with well-established analytical tools increasingly available [71-75], RNA-Seq is becoming an accessible tool that is superseding microarray in the research setting. We foresee that, with the aid of initiatives such as the SEquencing Quality Control (SEQC) consortium (the third phase of the FDA-led MAQC project) [76] and studies such as ours confirming its reliability, RNA-Seq will eventually also supersede microarrays in the clinical setting.

\section{Additional files}

Additional file 1: Table S1. Read statistics. "Proper pairs" are defined as reads where both pairs map to the reference genome with an inner distance between 60 and 160 bases. Table S2. Spearman correlation coefficients for each gene considered for comparison between Affymetrix microarray and Illumina RNA-Seq platforms. Table S3. Contingency table for breast cancer subtype classifiers (SCMs and SSPS) using Affymetrix microarray (AFFY) and Illumina RNA-Seq (RNASEQ) platforms. Table S4. Bootstrap analysis to assess the significance of the differences observed for Cohen's Kappa coefficients for subtype classifiers using Affymetrix microarray and Illumina RNA-Seq platforms.
Additional file 2: Figure S1. A and B: Box plots showing median level of expression on both Affymetrix microarray and Illumina RNA-Seq platforms for negatively and positively correlated genes.

Additional file 3: Figure S2. Correlation of gene expression levels between Affymetrix microarray and Illumina RNA-Seq platforms with respect to increasing quantiles of gene expression.

Additional file 4: Figure S3. Scatterplots reporting the point-to-point comparison of gene expression profiles measured by Affymetrix microarray and Illumina RNA-Seq platforms for each individual patient considered in the current study. Spearman correlation coefficient and p-value are provided below the plots. The solid line represents a linear regression of microarray values on the RNA-seq data while the dotted line has the equation $y=x$.

Additional file 5: Figure S4. Spearman correlation for the quantification of three clinically relevant genes (ER, PgR, and HER2) as defined by $\mathrm{HC}$ vs Affymetrix microarray and Illumina RNA-Seq, respectively.

Additional file 6: Figure S5. Bar plots representing the proportion of genes present in all signatures combined (3,663 unique genes in 27 signatures) with respect to their quantiles of expression for Affymetrix microarray (blue) and Illumina RNA-seq (red) platforms. The p-value reports the significance of the enrichment of signature genes with increasing quantiles of expression (Spearman's rank-based correlation).

Additional file 7: Figure S6. Bar plots representing, for each signature (27), the proportion of genes present in all signatures combined with respect to their quantiles of expression for Affymetrix microarray (blue) and Illumina RNA-seq (red) platforms. The p-value reports the significance of the enrichment of signature genes with increasing quantiles of expression (Spearman's rank-based correlation). Note that the SCMGENE and IRMODULE signatures contains few genes ( 3 and 6 genes, respectively) while the median signature size is 95 genes; for these small signatures, the $p$-value is expected to be large due to reduce sample size for the correlation analysis.

\section{Abbreviations}

RNA-Seq: RNA sequencing; BC: Breast cancer; GES: Gene expression signatures; fRMA: Frozen robust multiarray analysis; ER: Estrogen receptor; PgR: Progesterone receptor; HER2: Human epidermal growth factor receptor 2; IHC: Immunohistochemistry; FPKM: Fragments per kilobase per million mapped reads.

\section{Competing interests}

$\mathrm{SC}$ is inventor of the Genomic Grade Index (GGl); SC and DC are inventors of the immune signature; SC and HKB are inventors of the PIK3CA gene signature.

\section{Authors' contributions}

Conceived and designed the study: FD, DC, MS, GD, IM, PM, DV, SC, HKB. Performed the experiments: FD, MS, MM, RF. Provided pathology support: SR, LD. Analyzed the data: BCA, BD, GD, MS, DV, HKB. Wrote the paper: FD, BCA, $B D, S C, H K B$. Interpreted the data: all authors. All the authors read and approved the last version of the paper.

\section{Authors' information}

In addition to Debora Fumagalli, Alexis Blanchet-Cohen and David Brown being first authors and equal contributors, Christos Sotiriou and Benjamin Haibe-Kains are second author equal contributors.

\section{Acknowledgments}

We thank patients for having provided tissue and clinical data, and the Plan National Cancer (Belgium) for having supported the project. B. Haibe-Kains was supported by the Gattuso Slaight Personalized Cancer Medicine Fund at Princess Margaret Cancer Centre.

\section{Author details}

${ }^{1}$ Breast Cancer Translational Research Laboratory (BCTL), Institut Jules Bordet, Brussels, Belgium. ${ }^{2}$ Bioinformatics Core Facility, Institut de Recherches cliniques de Montréal, Montreal, QC, Canada. ${ }^{3}$ IRIBHM, Université Libre de Bruxelles, Campus Erasme, Brussels, Belgium. ${ }^{4}$ Department of Biostatistics and Epidemiology, Institut Gustave-Roussy, Villejuif, France. ${ }^{5}$ Paris-Sud University, Paris, France. ${ }^{6}$ Breast International Group, Brussels, Belgium. ${ }^{7}$ Department of 
Pathology, Institut Jules Bordet, Brussels, Belgium. ${ }^{8}$ Princess Margaret Cancer Centre, University Health Network, Toronto, ON, Canada. ${ }^{9}$ Medical Biophysics Department, University of Toronto, Toronto, ON, Canada.

Received: 5 August 2014 Accepted: 10 November 2014

Published: 21 November 2014

\section{References}

1. Van Hal NL, Vorst O, van Houwelingen AM, Kok EJ, Peijnenburg A, Aharoni A, van Tunen AJ, Keijer J: The application of DNA microarrays in gene expression analysis. J Biotechnol 2000, 78:271-280.

2. Perou CM, Sørlie T, Eisen MB, van de Rijn M, Jeffrey SS, Rees CA, Pollack JR, Ross DT, Johnsen H, Akslen LA, Fluge O, Pergamenschikov A, Williams C, Zhu SX, Lønning PE, Børresen-Dale AL, Brown PO, Botstein D: Molecular portraits of human breast tumours. Nature 2000, 406:747-752.

3. Peppercorn J, Perou CM, Carey LA: Molecular subtypes in breast cancer evaluation and management: divide and conquer. Cancer Invest 2008, 26:1-10.

4. Sørlie T, Perou CM, Tibshirani R, Aas T, Geisler S, Johnsen H, Hastie T, Eisen $M B$, van de Rijn M, Jeffrey SS, Thorsen T, Quist H, Matese JC, Brown PO, Botstein D, Lønning PE, Børresen-Dale AL: Gene expression patterns of breast carcinomas distinguish tumor subclasses with clinical implications. Proc Natl Acad Sci U S A 2001, 98:10869-10874.

5. Sotiriou C, Neo S-Y, McShane LM, Korn EL, Long PM, Jazaeri A, Martiat P, Fox $S B$, Harris AL, Liu ET: Breast cancer classification and prognosis based on gene expression profiles from a population-based study. Proc Natl Acad Sci U S A 2003, 100:10393-10398.

6. Goldhirsch A, Winer EP, Coates AS, Gelber RD, Piccart-Gebhart M, Thürlimann B, Senn $\mathrm{H}-$ J, Panel members: Personalizing the treatment of women with early breast cancer: highlights of the St Gallen International expert consensus on the primary therapy of early breast cancer 2013. Ann Oncol 2013, 24:2206-2223.

7. Desmedt C, Haibe-Kains B, Wirapati P, Buyse M, Larsimont D, Bontempi G, Delorenzi M, Piccart M, Sotiriou C: Biological processes associated with breast cancer clinical outcome depend on the molecular subtypes. Clin Cancer Res 2008, 14:5158-5165.

8. Wirapati P, Sotiriou C, Kunkel S, Farmer P, Pradervand S, Haibe-Kains B, Desmedt C, Ignatiadis M, Sengstag T, Schütz F, Goldstein DR, Piccart M, Delorenzi M: Meta-analysis of gene expression profiles in breast cancer: toward a unified understanding of breast cancer subtyping and prognosis signatures. Breast Cancer Res 2008, 10:R65.

9. Haibe-Kains B, Desmedt C, Loi S, Culhane AC, Bontempi G, Quackenbush J, Sotiriou C: A three-gene model to robustly identify breast cancer molecular subtypes. J Natl Cancer Inst 2012, 104:311-325.

10. Sorlie T, Tibshirani R, Parker J, Hastie T, Marron JS, Nobel A, Deng S, Johnsen H, Pesich R, Geisler S, Demeter J, Perou CM, Lønning PE, Brown PO, Børresen-Dale A-L, Botstein D: Repeated observation of breast tumor subtypes in independent gene expression data sets. Proc Natl Acad Sci U S A 2003, 100:8418-8423.

11. Hu Z, Fan C, Oh DS, Marron JS, He X, Qaqish BF, Livasy C, Carey LA, Reynolds E, Dressler L, Nobel A, Parker J, Ewend MG, Sawyer LR, Wu J, Liu Y, Nanda R, Tretiakova M, Ruiz Orrico A, Dreher D, Palazzo JP, Perreard L, Nelson E, Mone M, Hansen H, Mullins M, Quackenbush JF, Ellis MJ, Olopade $\mathrm{Ol}$, Bernard PS, et al: The molecular portraits of breast tumors are conserved across microarray platforms. BMC Genomics 2006, 7:96.

12. Parker JS, Mullins $M$, Cheang MCU, Leung S, Voduc D, Vickery T, Davies $S$, Fauron C, He X, Hu Z, Quackenbush JF, Stijleman IJ, Palazzo J, Marron JS, Nobel AB, Mardis E, Nielsen TO, Ellis MJ, Perou CM, Bernard PS: Supervised risk predictor of breast cancer based on intrinsic subtypes. J Clin Oncol 2009, 27:1160-1167.

13. Package Insert: Prosigna Breast Cancer Prognostic Gene Signature Assay; Version 01; 2013. REF LBL-C0223-01.

14. Veer $L$ V't, Dai $H$, van de Vijver MJ, He YD, Hart AAM, Mao M, Peterse HL, van der Kooy K, Marton MJ, Witteveen AT, Schreiber GJ, Kerkhoven RM, Roberts C, Linsley PS, Bernards R, Friend SH: Gene expression profiling predicts clinical outcome of breast cancer. Nature 2002, 415:530-536.

15. Paik S, Shak S, Tang G, Kim C, Baker J, Cronin M, Baehner FL, Walker MG, Watson D, Park T, Hiller W, Fisher ER, Wickerham DL, Bryant J, Wolmark N: A multigene assay to predict recurrence of tamoxifen-treated, node-negative breast cancer. N Engl J Med 2004, 351:2817-2826.
16. Sotiriou C, Wirapati $P$, Loi $S$, Harris A, Fox S, Smeds J, Nordgren H, Farmer P, Praz V, Haibe-Kains B, Desmedt C, Larsimont D, Cardoso F, Peterse H, Nuyten D, Buyse M, Van de Vijver MJ, Bergh J, Piccart M, Delorenzi M: Gene expression profiling in breast cancer: understanding the molecular basis of histologic grade to improve prognosis. J Natl Cancer Inst 2006, 98:262-272.

17. Filipits M, Rudas M, Jakesz R, Dubsky P, Fitzal F, Singer CF, Dietze O, Greil R, Jelen A, Sevelda P, Freibauer C, Müller V, Jänicke F, Schmidt M, Kölbl H, Rody A, Kaufmann M, Schroth W, Brauch H, Schwab M, Fritz P, Weber KE, Feder IS, Hennig G, Kronenwett R, Gehrmann M, Gnant M, EP Investigators: A new molecular predictor of distant recurrence in ER-positive, HER2negative breast cancer adds independent information to conventional clinical risk factors. Clin Cancer Res 2011, 17:6012-6020.

18. Carter SL, Eklund AC, Kohane IS, Harris LN, Szallasi Z: A signature of chromosomal instability inferred from gene expression profiles predicts clinical outcome in multiple human cancers. Nat Genet 2006, 38:1043-1048.

19. Teschendorff AE, Miremadi A, Pinder SE, Ellis IO, Caldas C: An immune response gene expression module identifies a good prognosis subtype in estrogen receptor negative breast cancer. Genome Biol 2007, 8:R157.

20. Farmer $P$, Bonnefoi $H$, Anderle $P$, Cameron $D$, Wirapati $P$, Wirapati $P$, Becette V, André S, Piccart M, Campone M, Brain E, Macgrogan G, Petit T, Jassem J, Bibeau F, Blot E, Bogaerts J, Aguet M, Bergh J, Iggo R, Delorenzi M: A stroma-related gene signature predicts resistance to neoadjuvant chemotherapy in breast cancer. Nat Med 2009, 15:68-74.

21. Desmedt C, Majjaj S, Kheddoumi N, Singhal SK, Haibe-Kains B, El Ouriaghli F, Chaboteaux C, Michiels S, Lallemand F, Journe F, Duvillier H, Loi S, Quackenbush J, Dekoninck S, Blanpain C, Lagneaux L, Houhou N, Delorenzi M, Larsimont D, Piccart M, Sotiriou C: Characterization and clinical evaluation of $\mathrm{CD} 10+$ stroma cells in the breast cancer microenvironment. Clin Cancer Res 2012, 18:1004-1014.

22. Loi S, Haibe-Kains B, Majjaj S, Lallemand F, Durbecq V, Larsimont D, Gonzalez-Angulo AM, Pusztai L, Symmans WF, Bardelli A, Ellis P, Tutt ANJ, Gillett CE, Hennessy BT, Mills GB, Phillips WA, Piccart MJ, Speed TP, McArthur GA, Sotiriou C: PIK3CA mutations associated with gene signature of low mTORC1 signaling and better outcomes in estrogen receptor-positive breast cancer. Proc Natl Acad Sci U S A 2010, 107:10208-10213.

23. Saal LH, Johansson P, Holm K, Gruvberger-Saal SK, She Q-B, Maurer M, Koujak S, Ferrando AA, Malmström P, Memeo L, Isola J, Bendahl P-O, Rosen $\mathrm{N}$, Hibshoosh $\mathrm{H}$, Ringnér M, Borg A, Parsons R: Poor prognosis in carcinoma is associated with a gene expression signature of aberrant PTEN tumor suppressor pathway activity. Proc Natl Acad Sci U S A 2007, 104:7564-7569.

24. Creighton CJ, Casa A, Lazard Z, Huang S, Tsimelzon A, Hilsenbeck SG, Osborne CK, Lee AV: Insulin-like growth factor-I activates gene transcription programs strongly associated with poor breast cancer prognosis. J Clin Oncol 2008, 26:4078-4085.

25. Majumder PK, Febbo PG, Bikoff R, Berger R, Xue Q, McMahon LM, Manola J, Brugarolas J, McDonnell TJ, Golub TR, Loda M, Lane HA, Sellers WR: mTOR inhibition reverses Akt-dependent prostate intraepithelial neoplasia through regulation of apoptotic and HIF-1-dependent pathways. Nat Med 2004, 10:594-601.

26. Creighton CJ, Hilger AM, Murthy S, Rae JM, Chinnaiyan AM, El-Ashry D: Activation of mitogen-activated protein kinase in estrogen receptor alpha-positive breast cancer cells in vitro induces an in vivo molecular phenotype of estrogen receptor alpha-negative human breast tumors. Cancer Res 2006, 66:3903-3911.

27. Bild AH, Yao G, Chang JT, Wang Q, Potti A, Chasse D, Joshi M-B, Harpole D, Lancaster JM, Berchuck A, Olson JA Jr, Marks JR, Dressman HK, West M, Nevins JR: Oncogenic pathway signatures in human cancers as a guide to targeted therapies. Nature 2006, 439:353-357.

28. Mortazavi A, Williams BA, McCue K, Schaeffer L, Wold B: Mapping and quantifying mammalian transcriptomes by RNA-Seq. Nat Methods 2008, 5:621-628.

29. Sultan M, Schulz MH, Richard H, Magen A, Klingenhoff A, Scherf M, Seifert M, Borodina T, Soldatov A, Parkhomchuk D, Schmidt D, O'Keeffe S, Haas S, Vingron M, Lehrach $H$, Yaspo M-L: A global view of gene activity and alternative splicing by deep sequencing of the human transcriptome. Science 2008, 321:956-960.

30. Wang Z, Gerstein M, Snyder M: RNA-Seq: a revolutionary tool for transcriptomics. Nat Rev Genet 2009, 10:57-63. 
31. Ozsolak F, Milos PM: RNA sequencing: advances, challenges and opportunities. Nat Rev Genet 2011, 12:87-98.

32. Malone $\mathrm{JH}$, Oliver B: Microarrays, deep sequencing and the true measure of the transcriptome. BMC Bio/ 2011, 9:34.

33. Nagalakshmi U, Wang Z, Waern K, Shou C, Raha D, Gerstein M, Snyder M: The transcriptional landscape of the yeast genome defined by RNA sequencing. Science 2008, 320:1344-1349.

34. Marioni JC, Mason CE, Mane SM, Stephens M, Gilad Y: RNA-seq: an assessment of technical reproducibility and comparison with gene expression arrays. Genome Res 2008, 18:1509-1517.

35. Fu X, Fu N, Guo S, Yan Z, Xu Y, Hu H, Menzel C, Chen W, Li Y, Zeng R, Khaitovich P: Estimating accuracy of RNA-Seq and microarrays with proteomics. BMC Genomics 2009, 10:161.

36. Montgomery SB, Sammeth M, Gutierrez-Arcelus M, Lach RP, Ingle C, Nisbett J, Guigo R, Dermitzakis ET: Transcriptome genetics using second generation sequencing in a Caucasian population. Nature 2010, 464:773-777.

37. Pickrell JK, Marioni JC, Pai AA, Degner JF, Engelhardt BE, Nkadori E, Veyrieras J-B, Stephens M, Gilad Y, Pritchard JK: Understanding mechanisms underlying human gene expression variation with RNA sequencing. Nature 2010, 464:768-772.

38. Bradford JR, Hey Y, Yates T, Li Y, Pepper SD, Miller CJ: A comparison of massively parallel nucleotide sequencing with oligonucleotide microarrays for global transcription profiling. BMC Genomics 2010, 11:282.

39. Liu S, Lin L, Jiang P, Wang D, Xing Y: A comparison of RNA-Seq and highdensity exon array for detecting differential gene expression between closely related species. Nucleic Acids Res 2011, 39:578-588.

40. Nookaew I, Papini M, Pornputtapong N, Scalcinati G, Fagerberg L, Uhlén M, Nielsen J: A comprehensive comparison of RNA-Seq-based transcriptome analysis from reads to differential gene expression and cross-comparison with microarrays: a case study in Saccharomyces cerevisiae. Nucleic Acids Res 2012, 40:10084-10097.

41. Sekhon RS, Briskine R, Hirsch CN, Myers CL, Springer NM, Buell CR, de Leon N, Kaeppler SM: Maize gene atlas developed by RNA sequencing and comparative evaluation of transcriptomes based on RNA sequencing and microarrays. PLoS One 2013, 8:e61005.

42. Xu X, Zhang Y, Williams J, Antoniou E, McCombie WR, Wu S, Zhu W, Davidson NO, Denoya P, Li E: Parallel comparison of Illumina RNA-Seq and Affymetrix microarray platforms on transcriptomic profiles generated from 5-aza-deoxy-cytidine treated HT-29 colon cancer cells and simulated datasets. BMC Bioinformatics 2013, 14(Suppl 9):S1.

43. Sotiriou C, Pusztai L: Gene-expression signatures in breast cancer. N Engl J Med 2009, 360:790-800.

44. ArrayAnalysis.org. In http://www.arrayanalysis.org/.

45. McCall MN, Bolstad BM, Irizarry RA: Frozen robust multiarray analysis (fRMA). Biostatistics 2010, 11:242-253.

46. R Development Core Team: R: A Language and Environment for Statistical Computing; 2011. ISBN 3-900051-07-0.

47. Li Q, Birkbak NJ, Gyorffy B, Szallasi Z, Eklund AC: Jetset: selecting the optimal microarray probe set to represent a gene. BMC Bioinformatics 2011, 12:474.

48. Durinck S, Moreau Y, Kasprzyk A, Davis S, De Moor B, Brazma A, Huber W: BioMart and Bioconductor: a powerful link between biological databases and microarray data analysis. Bioinformatics 2005, 21:3439-3440.

49. European Genome-phenome Archive. In https://www.ebi.ac.uk/ega.

50. Andrews S: FastQC A Quality Control tool for High Throughput Sequence Data. In 2014. http://www.bioinformatics.babraham.ac.uk/projects/fastqc/.

51. Barnett DW, Garrison EK, Quinlan AR, Strömberg MP, Marth GT: BamTools: a $\mathrm{C}++\mathrm{API}$ and toolkit for analyzing and managing BAM files. Bioinformatics 2011, 27:1691-1692.

52. Picard. In 2014. http://picard.sourceforge.net.

53. Garber M, Grabherr MG, Guttman M, Trapnell C: Computational methods for transcriptome annotation and quantification using RNA-seq. Nat Methods 2011, 8:469-477

54. Bioconductor-genefu. In http://www.bioconductor.org/packages/release/ bioc/html/genefu.html.

55. Ignatiadis M, Singhal SK, Desmedt C, Haibe-Kains B, Criscitiello C, Andre F, Loi S, Piccart M, Michiels S, Sotiriou C: Gene modules and response to neoadjuvant chemotherapy in breast cancer subtypes: a pooled analysis. J Clin Oncol 2012, 30:1996-2004.

56. Culhane AC, Schröder MS, Sultana R, Picard SC, Martinelli EN, Kelly C, Haibe-Kains B, Kapushesky M, St Pierre A-A, Flahive W, Picard KC,
Gusenleitner D, Papenhausen G, O'Connor N, Correll M, Quackenbush J: GeneSigDB:

a manually curated database and resource for analysis of gene expression signatures. Nucleic Acids Res 2012, 40(Database issue):D1060-D1066.

57. Gentleman R: Reproducible research: a bioinformatics case study. Stat Appl Genet Mol Biol 2005, 4:1544-6115.

58. Subramaniam S, Hsiao G: Gene-expression measurement: variance-modeling considerations for robust data analysis. Nat Immunol 2012, 13:199-203.

59. Harris L, Fritsche H, Mennel R, Norton L, Ravdin P, Taube S, Somerfield MR, Hayes DF, Bast RC Jr, American Society of Clinical Oncology: American society of clinical oncology 2007 update of recommendations for the use of tumor markers in breast cancer. J Clin Oncol 2007, 25:5287-5312.

60. Hammond MEH, Hayes DF, Dowsett M, Allred DC, Hagerty KL, Badve S, Fitzgibbons PL, Francis G, Goldstein NS, Hayes M, Hicks DG, Lester S, Love R, Mangu PB, McShane L, Miller K, Osborne CK, Paik S, Perlmutter J, Rhodes A, Sasano H, Schwartz JN, Sweep FCG, Taube S, Torlakovic EE, Valenstein P, Viale G, Visscher D, Wheeler T, Williams RB, et al: American Society of Clinical Oncology/College of American Pathologists guideline recommendations for immunohistochemical testing of estrogen and progesterone receptors in breast cancer (unabridged version). Arch Pathol Lab Med 2010, 134:e48-e72.

61. Wolff AC, Hammond MEH, Hicks DG, Dowsett M, McShane LM, Allison KH, Allred DC, Bartlett JMS, Bilous M, Fitzgibbons P, Hanna W, Jenkins RB, Mangu PB, Paik S, Perez EA, Press MF, Spears PA, Vance GH, Viale G, Hayes DF: Recommendations for human epidermal growth factor receptor 2 testing in breast cancer: American society of clinical oncology/College of American Pathologists clinical practice guideline update. Arch Pathol Lab Med 2014, 138:241-256.

62. Cardoso F, Van't Veer L, Rutgers E, Loi S, Mook S, Piccart-Gebhart MJ: Clinical application of the 70-gene profile: the MINDACT trial. J Clin Oncol 2008, 26:729-735.

63. Sparano JA: TAILORx: trial assigning individualized options for treatment (Rx). Clin Breast Cancer 2006, 7:347-350.

64. MAQC Consortium, Shi L, Reid LH, Jones WD, Shippy R, Warrington JA Baker SC, Collins PJ, de Longueville F, Kawasaki ES, Lee KY, Luo Y, Sun YA Willey JC, Setterquist RA, Fischer GM, Tong W, Dragan YP, Dix DJ, Frueh FW, Goodsaid FM, Herman D, Jensen RV, Johnson CD, Lobenhofer EK, Puri RK, Schrf U, Thierry-Mieg J, Wang C, Wilson M, et al: The MicroArray Quality Control (MAQC) project shows inter- and intraplatform reproducibility of gene expression measurements. Nat Biotechnol 2006, 24:1151-1161.

65. Guo Y, Sheng Q, Li J, Ye F, Samuels DC, Shyr Y: Large scale comparison of gene expression levels by microarrays and RNAseq using TCGA data. PLoS One 2013, 8:e71462

66. Roepman P, Horlings HM, Krijgsman O, Kok M, Bueno-de-Mesquita JM, Bender R, Linn SC, Glas AM, van de Vijver MJ: Microarray-based determination of estrogen receptor, progesterone receptor, and HER2 receptor status in breast cancer. Clin Cancer Res 2009, 15:7003-7011.

67. Wesseling J, Tinterri C, Sapino A, Zanconati F, Lutke Holzik M, Nguyen B, Deck KB, Querzoli P, Perin T, Giardina C, Seitz G, Guinebretiere J-M, Barone J, Watanabe T, De Snoo F, Stork-Sloots L, Cusumano P: Central review of discordant samples for microarray-based ER, PR, and HER2 and local IHC/FISH assessment worldwide from 827 patients [abstract]. J Clin Oncol 2012, 30: suppl 27; abstr 11

68. Li Q, Eklund AC, Juul N, Haibe-Kains B, Workman CT, Richardson AL, Szallasi Z, Swanton C: Minimising immunohistochemical false negative ER classification using a complementary 23 gene expression signature of ER status. PLoS One 2010, 5:e15031.

69. Kamalakaran S, Lezon-Geyda K, Varadan V, Banerjee N, Lannin D, Rizack T, Sikov W, Abu-Khalaf M, Janevski A, Harris L: Evaluation of ER/PR and HER2 status by RNA sequencing in tissue core biopsies from preoperative clinical trial specimens [abstract]. J Clin Oncol 2011, 29: suppl 27; abstr 46

70. Lusa L, McShane LM, Reid JF, De Cecco L, Ambrogi F, Biganzoli E, Gariboldi M, Pierotti MA: Challenges in projecting clustering results across gene expression-profiling datasets. J Natl Cancer Inst 2007, 99:1715-1723.

71. Auer PL, Doerge RW: Statistical design and analysis of RNA sequencing data. Genetics 2010, 185:405-416.

72. Bullard $\mathrm{JH}$, Purdom E, Hansen KD, Dudoit S: Evaluation of statistical methods for normalization and differential expression in mRNA-Seq experiments. BMC Bioinformatics 2010, 11:94.

73. Zheng $W$, Chung LM, Zhao H: Bias detection and correction in RNASequencing data. BMC Bioinformatics 2011, 12:290. 
74. Dillies M-A, Rau A, Aubert J, Hennequet-Antier C, Jeanmougin M, Servant N, Keime C, Marot G, Castel D, Estelle J, Guernec G, Jagla B, Jouneau L, Laloë D, Le Gall C, Schaëffer B, Le Crom S, Guedj M, Jaffrézic F, French StatOmique Consortium: A comprehensive evaluation of normalization methods for Illumina high-throughput RNA sequencing data analysis. Brief Bioinformatics 2013, 14:671-683.

75. Goecks J, Nekrutenko A, Taylor J, Galaxy Team: Galaxy: a comprehensive approach for supporting accessible, reproducible, and transparent computational research in the life sciences. Genome Biol 2010, 11:R86.

76. MAQC Project. In http://www.fda.gov/ScienceResearch/BioinformaticsTools/ MicroarrayQualityControlProject/.

doi:10.1186/1471-2164-15-1008

Cite this article as: Fumagalli et al:: Transfer of clinically relevant gene expression signatures in breast cancer: from Affymetrix microarray to Illumina RNA-Sequencing technology. BMC Genomics 2014 15:1008.

\section{Submit your next manuscript to BioMed Central and take full advantage of:}

- Convenient online submission

- Thorough peer review

- No space constraints or color figure charges

- Immediate publication on acceptance

- Inclusion in PubMed, CAS, Scopus and Google Scholar

- Research which is freely available for redistribution 